\title{
Erratum to: Treatment patterns and outcomes in the prophylaxis of chemotherapy-induced (febrile) neutropenia with biosimilar filgrastim (the MONITOR-GCSF study)
}

\author{
Pere Gascón $^{1}$ - Matti Aapro ${ }^{2}$ Heinz Ludwig ${ }^{3}$ - Carsten Bokemeyer ${ }^{4}$. \\ Mario Boccadoro $^{5} \cdot$ Matthew Turner $^{6} \cdot$ Kris Denhaerynck $^{7} \cdot$ Karen MacDonald $^{7}$. \\ Ivo Abraham ${ }^{7,8}$
}

Published online: 19 October 2015

(C) Springer-Verlag Berlin Heidelberg 2015

\section{Erratum to: Support Care Cancer \\ DOI 10.1007/s00520-015-2861-z}

Table 1 footer definition for "advanced disease" (footer d) should read: $\mathrm{d}$ Stage 4 (Stage 3 if multiple myeloma) and prior chemo in metastatic setting

Ivo Abraham

abraham@pharmacy.arizona.edu

1 Division of Medical Oncology, Department of

Hematology-Oncology, Hospital Clínic de Barcelona, University of Barcelona, Barcelona, Spain

2 Institut Multidisciplinaire d'Oncologie, Clinique de Genolier, Genolier, Switzerland

3 Medizinische Abteilung I-Onkologie und Haematologie, Wilhelminenspital, Wien, Austria
4 Universitaetsklinikum Hamburg Eppendorf, Hamburg, Germany

5 Dipartimento di Oncologia e Ematologia, Azienda Ospedaliero, Universitaria S. Giovanni Battista di Torino, Torino, Italy

6 Sandoz Biopharmaceuticals, Holzkirchen, Germany

7 Matrix45, Tucson, AZ, USA

8 Center for Health Outcomes and PharmacoEconomic Research, University of Arizona, Drachman Hall B306H, 1295 N Martin, Tucson, AZ 85721, USA 